\title{
Single camera 3D planar Doppler velocity measurements, using two frequency Planar Doppler Velocimetry (2v-PDV) and imaging fibre bundles
}

\author{
Tom O. Charrett, Helen D. Ford and Ralph P. Tatam \\ Optical Sensors Group, Centre for Photonics and Optical Engineering, School of Engineering, Cranfield University, \\ Bedfordshire, MK43 OAL, UK
}

\begin{abstract}
A modified Planar Doppler Velocimetry (PDV) technique, two frequency PDV (2v-PDV), is described that allows measurements of the velocity field over a plane defined by a laser light sheet using sequential illumination of the flow with two closely separated ( 1GHz) frequencies of laser light. This allows a common-path imaging head to be used containing a single CCD camera instead of the usual camera pair. The problem of image misalignment is now avoided and the polarisation sensitivity of the beam splitter used in two camera imaging heads is also removed. Cost efficiency is improved by the simplification of the system. This paper describes the development of a $2 \mathrm{v}$-PDV system using a continuous wave argon ion laser capable of making time-averaged velocity measurements. Initially a single velocity component system was constructed using acousto-optic modulators to produce the two illumination frequencies required. The system was then expanded to make 3D velocity measurements using a single CCD camera and multiple coherent imaging fibre bundles. Measurements were made on the rotating disc, in order to assess error level in the measurements, and on a seeded axisymmetric air jet. A method of improving the sensitivity of the $2 v$-PDV system is demonstrated by using both the rising and falling slopes of the iodine absorption line. Reductions in the error levels of velocity measurements of approximately $40 \%$ can be achieved using this increased sensitivity method.
\end{abstract}

\section{Introduction}

Planar Doppler Velocimetry (PDV) $)^{1-4}$, also know as Doppler Global Velocimetry (DGV), is a flow measurement technique that provides velocity information over a plane defined by a laser light sheet. PDV relies upon measuring the Doppler frequency shift of light scattered from particles entrained in the flow. As PDV relies upon the Doppler shift a single observation direction can measure a single component of velocity, as shown in figure 1 . The use of imaging fibre bundles ${ }^{5}$ allows several observation directions, and thus velocity components, to be measured simultaneously by spatially multiplexing three or more images of the flow field onto a single CCD camera. The optical frequency shift, $\boldsymbol{\Delta v}$, is given by the Doppler equation:

$$
\Delta v=\frac{v(\hat{o}-\hat{i}) \cdot V}{c}
$$

where $\boldsymbol{v}$ is the optical frequency, $\hat{\boldsymbol{o}}$ and $\hat{\boldsymbol{i}}$ are unit vectors in the observation and illumination directions respectively, $\boldsymbol{V}$ is the velocity vector and $c$ is the free space speed of light. The optical frequency of light scattered from each particle in the seeded flow experiences a Doppler shift, which is linearly related to the velocity of the particle at that point in the flow. In PDV, a region of the illuminated flow is imaged, through a glass cell usually containing iodine vapor, onto the active area of a CCD camera. Iodine has numerous narrow absorption lines over a large part of the visible spectrum ${ }^{6}$. The laser frequency is chosen to coincide with one such line and hence the optical intensity at any position in the camera image is a function of the Doppler shift experienced at the corresponding position in the flow, via the frequency-dependent iodine absorption. 


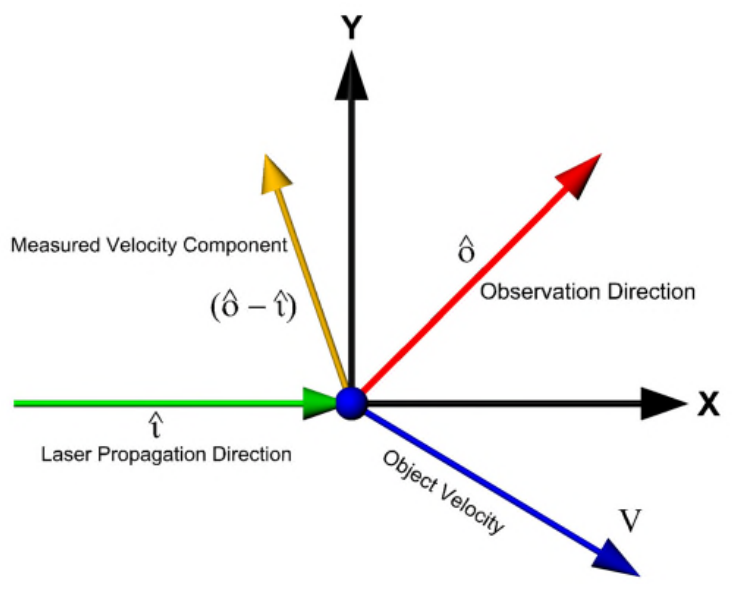

Figure 1. The relationship of laser illumination direction and observation direction to the measured velocity component determined from the Doppler equation.

of interest to a single detector head reduces the number of imaging heads needed and simplifies the system. intensity profile of the illuminating laser sheet (typically Gaussian), spatial variations of the seeding density within the flow, and diffraction fringes caused by imperfections in the optical surfaces. These variations are generally of similar amplitude to those resulting from absorption in the iodine cell, and can obscure the information about flow velocity that is contained within the camera image. It is therefore usual to amplitude-divide the image beam onto two cameras; from one of the two imaging paths the iodine cell is omitted, and the resulting image acts as a reference to normalize the signal image carrying the velocity information.

Viewing the flow from a single observation direction allows a single component of the flow velocity to be measured; therefore to make three-dimensional measurements a minimum of three viewing directions are necessary. Conventional methods use a separate PDV imaging head (two cameras and an iodine cell each) to measure each of the three velocity components. Using imaging fibre bundles to port multiple views of the region

The intensity over a PDV image is affected by the

\section{Two frequency Planar Doppler Velocimetry (2v-PDV)}

In the two-frequency Planar Doppler Velocimetry $(2 v-P D V)^{7 ; 8}$ technique the signal and reference images are acquired sequentially, on the same CCD camera by the use of two illumination frequencies.

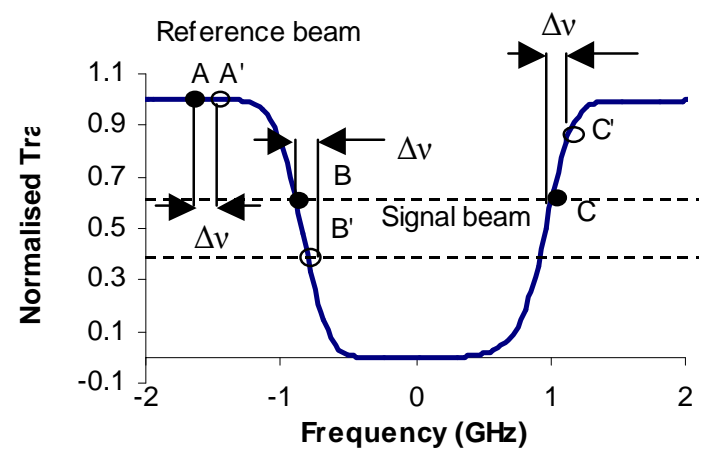

Figure 2. Relative positions of the laser frequency, and the shifted frequency on a typical absorption feature for $2 v$-PDV ( $A$ and $B / C$ denote the position of the illumination frequencies and $A^{\prime}$ and $B^{\prime} / C^{\prime}$ the Doppler shifted frequency.)
There are two approaches to the positioning of the illuminations frequencies relative to the iodine absorption line. In the first a signal and reference image are captured similar to conventional PDV. The emission wavelength of the laser is tuned just off the low frequency side of an absorption line. The output beam is first frequency up shifted to lie in a zero absorption region of the iodine transfer function (figure 2, point A). A reference image is then acquired. The frequency is then downshifted to lie approximately midway (50\%) on the iodine cell transfer function (figure 2, point $\mathrm{B}$ ), and a signal image is acquired.

The second approach increases the sensitivity of the system by tuning the two frequencies to the positions shown as $\mathrm{B}$ and $\mathrm{C}$ in figure 2 . With one source tuned onto the falling slope and the other on the rising slope, a constant Doppler shift will result in the further attenuation of one image to a lower signal level and the rise in the signal level in the other image. Dividing the difference of the images by the sum, and taking into account any difference in the gradients will give a result that has approximately double the sensitivity of the current PDV methods.

The optical frequency difference is $\sim 700-1000 \mathrm{MHz}$, which is sufficiently small that there will be no change in the scattering for the size of particles typically used; 0.2-5 $\mu \mathrm{m}$ diameter. Exact alignment of the reference and signal 
images on the active area of the camera is automatic. This means that the pixel-matching problem that affects conventional PDV is avoided. For example Thorpe et $\mathrm{al}^{9}$ assess the impact of image misalignment, on the velocity field of a rotating disc, and for an image misalignment of 0.1 pixels, they estimate this error to be $\pm 5 \mathrm{~ms}^{-1}$.

The method also eliminates the polarization sensitivity of the split ratio of the beam splitter used in two-camera systems ${ }^{10}$.Ideally the beam splitter used in a conventional PDV imaging head would split the incoming light 50:50 between the signal and reference cameras with no variation for different polarizations of light. However even the 'non-polarizing' beam splitters typically used retain a slight sensitivity to polarization, typically quoted as $\pm 3 \%$ variation in the split ratio for $\mathrm{S}$ and $\mathrm{P}$ polarized light, leading to typical velocity errors of $\pm 7 \mathrm{~ms}^{-1}$.

A two-wavelength method has been described by Arnette et al ${ }^{11}$, using two laser lines (532 and $618 \mathrm{~nm}$ ) and a colour CCD to capture signal and reference images simultaneously. Muller et al ${ }^{12}$ described a similar method using a frequency modulated Distributed Bragg Reflector (DBR) laser diode $(852.6 \mathrm{~nm}, 100 \mathrm{~mW}$ ) and a Caesium absorption cell to capture three sequential images at three wavelengths for a single velocity component.

\section{Development of a CW two frequency PDV (2v-PDV) system}

\section{A. Single velocity component measurements}

Initially the system was set-up to measure a single velocity component (figure 3 ). A continuous wave (CW) single frequency argon ion laser, operating at a wavelength of $515.5 \mathrm{~nm}$, was used as a light source, allowing time averaged velocity measurements to be made. The frequency shift available with this arrangement only allowed the use of the first of the $2 v$-PDV approaches, where signal and reference images, similar to conventional PDV, are captured.

The optical frequency of this light source was altered to form the reference and signal beams using a combination of two acousto-optic modulators (AOM). The generation of both the reference beam and the signal beam is shown in the detail of the two-frequency beam generator. The signal beam is generated when AOM 1 is switched on and AOM 2 is switched off, providing an up-shift of $520 \mathrm{MHz}$, when AOM 2 is on and AOM 1 off the reference beam is generated this has a $180 \mathrm{MHz}$ downshift in the frequency.

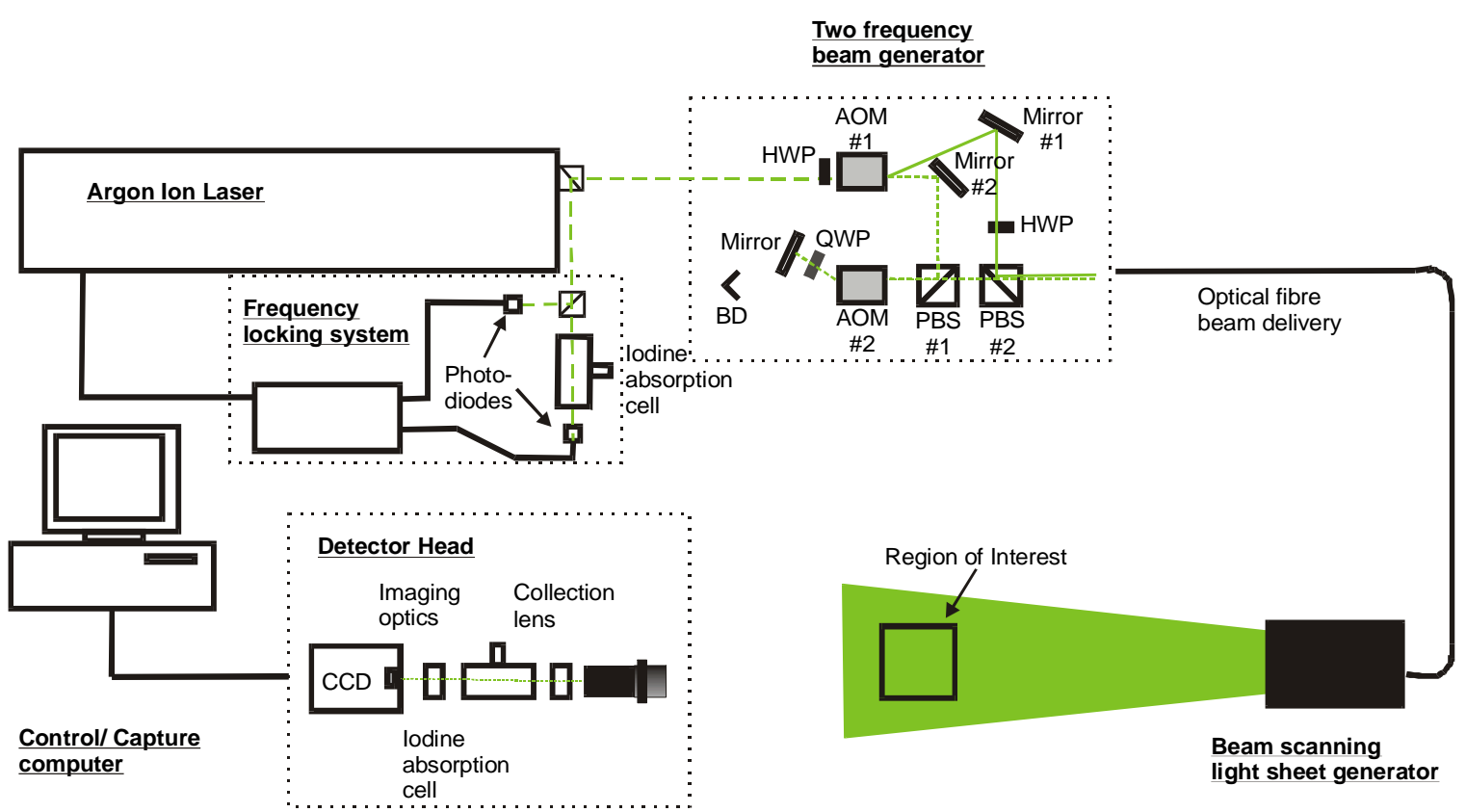

Figure 3. Schematic of the experimental arrangement for the single component measurements including detail of the two-frequency beam generator showing the generation of both the signal and reference beams.

HWP - $\lambda / 2$ plate; AOM - acousto-optic modulator; PBS - polarising beam splitter, QWP - $\lambda / 4$ plate; BD beam dump. Reference beam path Signal beam path - Un-shifted laser beam 
Both beams leave the beam generator and are coupled into an optical fibre. The beams are then delivered to a prism-scanning device ${ }^{13}$. This scans the collimated beam rapidly across the region of interest, resulting in an ideal 'top-hat' intensity profile of the generated light sheet. The desired illumination frequency can then be selected by toggling on/off the two AOMs.

A frequency locking system, shown in figure 3, is used to control the laser's fundamental frequency, and hence the positions of the two beams on the iodine absorption line. This consists of an iodine cell, signal and reference photodiodes and locking electronics which adjusts the laser etalon temperature to ensure that the laser frequency is stable, based upon the transmission through this cell.

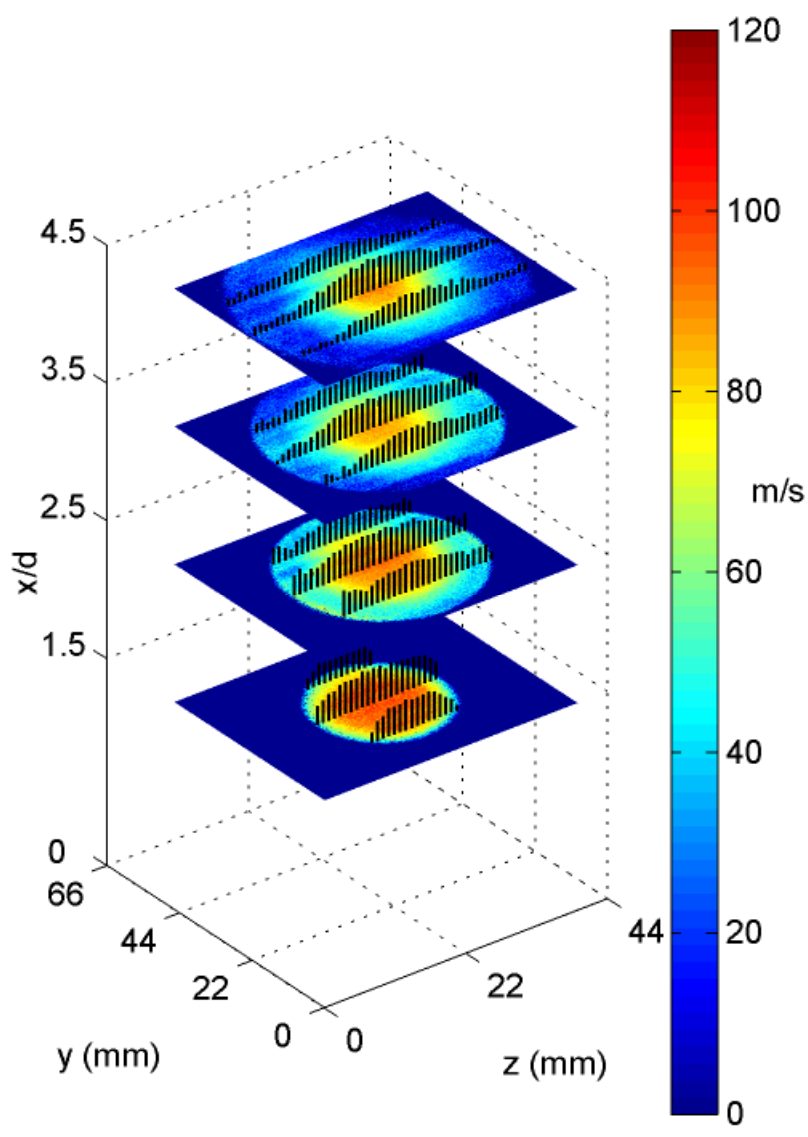

Figure 4. The velocity field of an axisymmetric air jet made using $2 v$-PDV. Measurements were taken at 1.5 , 2.5, 3.5 and 4.5 nozzle diameters downstream from the nozzle. Overlaid are vectors showing the magnitude of the velocity at various points (arrow heads have been removed for clarity)
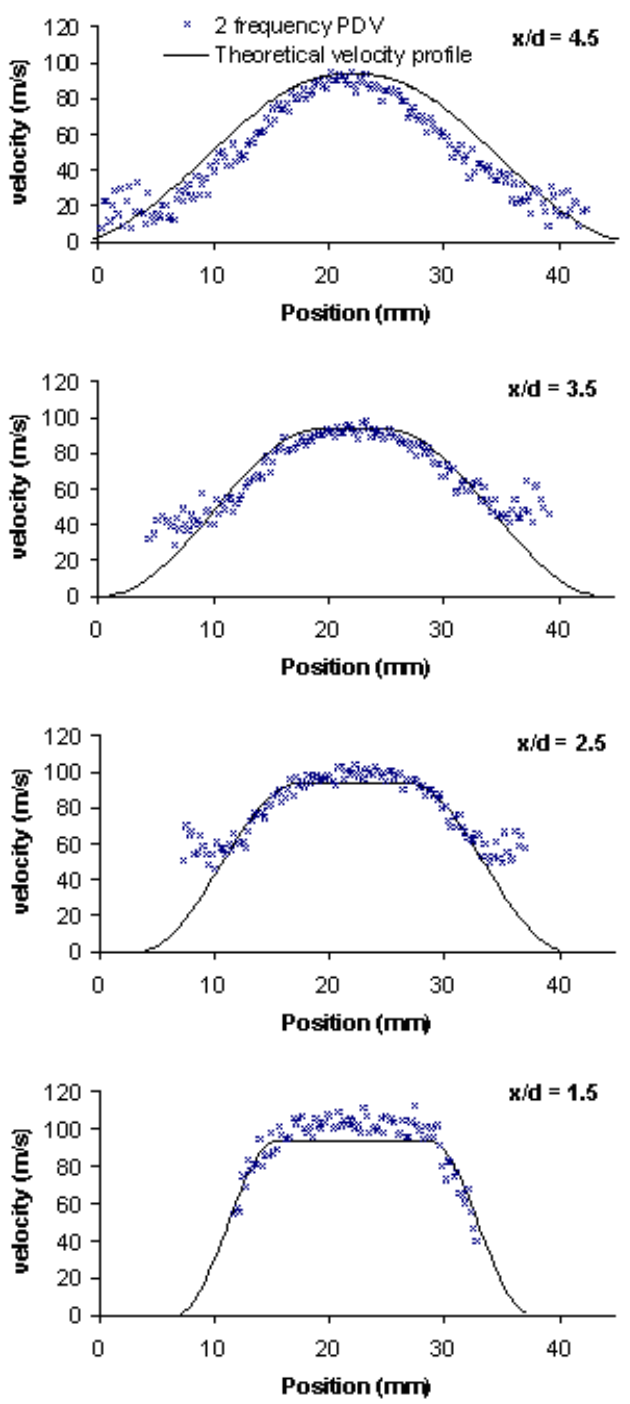

Figure 5. Profiles taken through the center of each slice, (crosses - experimental values, solid - theoretical velocity profile) 
The detector head images the region of interest in the flow using a standard SLR camera lens and a signal image and a reference image are captured under the appropriate illumination selected by toggling the AOMs on/off. Measurements were made on a seeded air jet to provide conditions similar to the techniques intended applications on seeded airflows. The jet used was an axis-symmetric air jet, with a $20 \mathrm{~mm}$ diameter smooth contraction nozzle that was seeded using a smoke generator producing seed particles in the $0.2-0.3 \mu \mathrm{m}$ diameter range. The jet has a theoretical exit velocity of $94 \mathrm{~ms}^{-1}$, which was calculated by measuring the nozzle pressure ratio. Figure 4 shows the main velocity component of this jet at various positions from the nozzle ${ }^{7}$ as measured using the $2 v$-PDV arrangement and figure 5 show profiles taken through the center of each slice compared with the theoretical velocity profile for the jet.

\section{B. Extension to multiple velocity components and 3d 2v-PDV measurements}

The system was then extended to measure multiple velocity components in the plane defined by the light sheet, using imaging fibre bundles 5 as the input into the detector head. Views of the region of interest are ported to the detector head using a coherent array of fibres that is split into four channels (figure 6). Each channel has 600x500 fibres that are $8 \mu \mathrm{m}$ in diameter and positioned at $10 \mu \mathrm{m}$ centers. These views are combined at the detector head, with each occupying a quarter of the CCD image (figure 7). An example of the image formed is shown in figure 8 . This is a view of a calibration target used to de-warp the views to a common view and determine the observation directions for each view ${ }^{14}$. Figure 9 shows the views after this de-warping process; here all four views have been overlaid for demonstration purposes. A similar multi-branch imaging fibre bundles system has been described by Willert et al ${ }^{15}$.

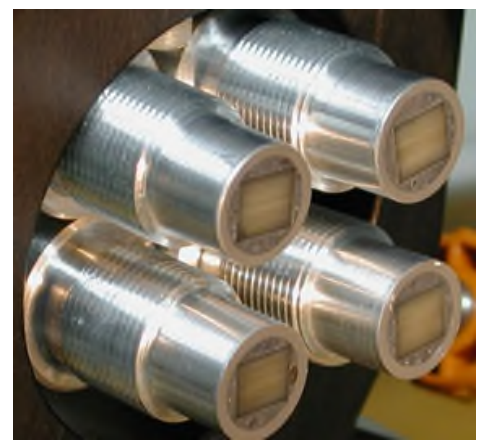

Figure 6. The individual arms of the imaging fibre bundles.

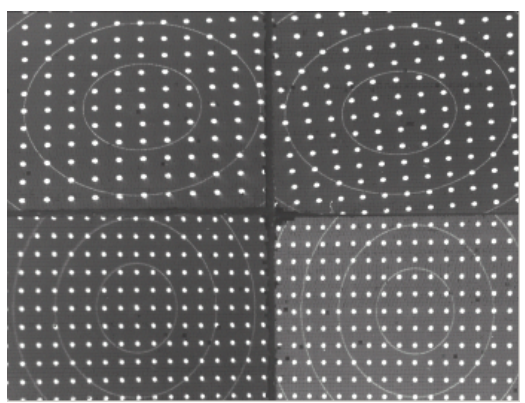

Figure 8. An example image of a view through the imaging bundles of a calibration target (field of view $\sim 100 \times 100 \mathrm{~mm}$ )

In order to measure all three components of velocity it is necessary to view from at least three directions, however the imaging fibre bundle in use has four channels. Here all four were used in the measurements. This is because we have found that the use of a fourth measured velocity component can significantly reduce the errors in the conversion from non-orthogonal measured velocity components to orthogonal velocity components. This will be discussed in more detail in a forthcoming publication.

American Institute of Aeronautics and Astronautics 
The arrangement of the beam generator, shown in figure 3, was very light inefficient and combined with the use of the imaging fibre bundles, resulted in low scattered light levels being collected. The experimental arrangement was modified to allow greater illumination power; this new arrangement is shown in figure 10. Here the laser frequency is selected by tuning the laser etalon voltage and is then stabilized using the locking system described above. The frequency of the locking beam is shifted by $260 \mathrm{MHz}$ so that the locking system can still operate if the laser is located at $100 \%$ transmission. The locking beam will be shifted onto the absorption line so that any frequency fluctuations will result in a transmission fluctuation seen by the photodiodes and can be corrected. Although this arrangement increased the illumination power the images are now captured with a separation of minutes rather than seconds, although for the time averaged measurements this has not been a problem.

As well as providing increased beam powers, it is now possible to tune both beam frequencies to coincide with the absorption line, one on the falling slope and the other on the rising slope allowing the increased sensitivity scheme described above to be used.

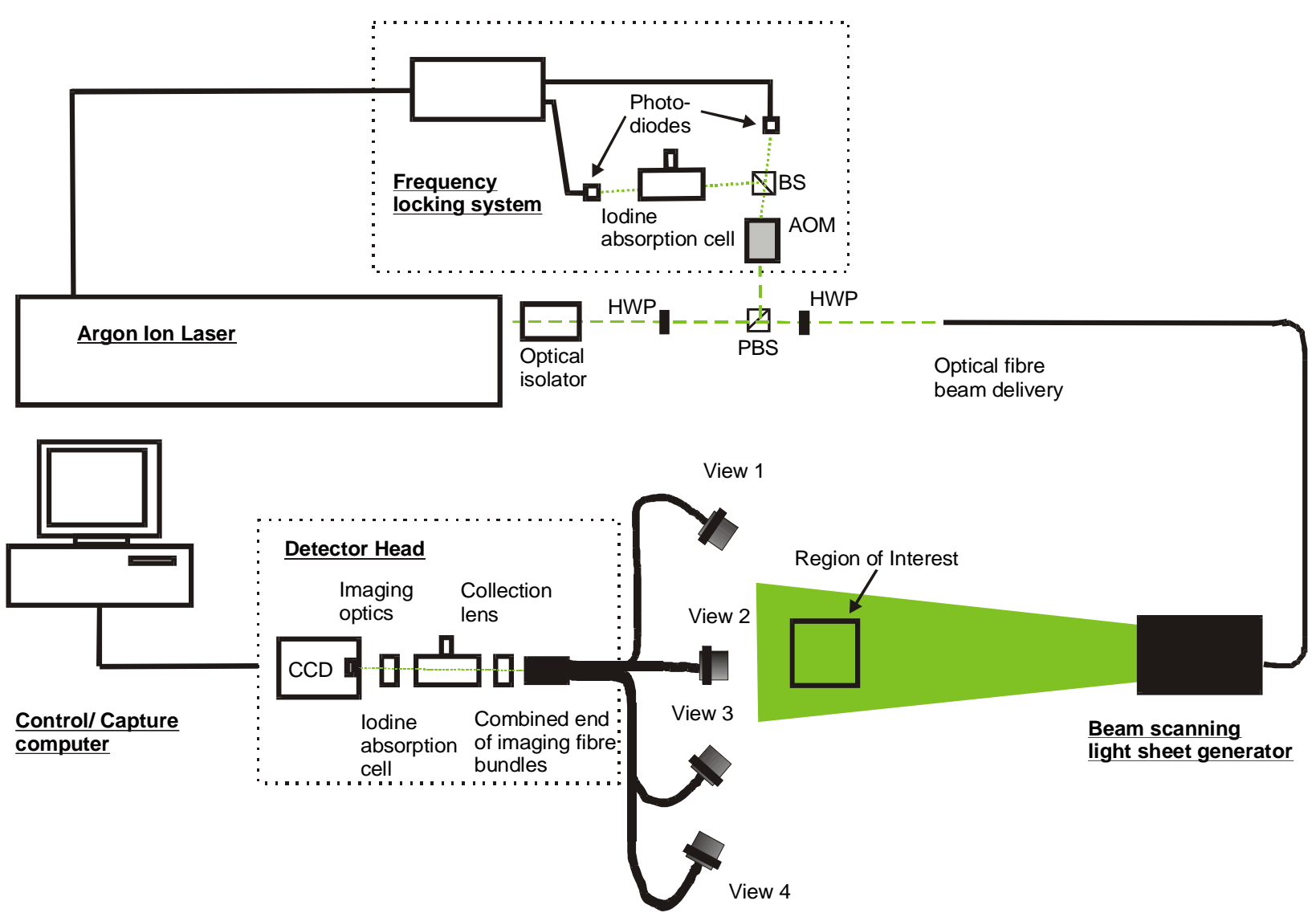

Figure 10. Schematic showing the experimental arrangement used for the three dimensional velocity measurements.

HWP - $\lambda / 2$ plate; AOM - acousto-optic modulator; PBS - polarizing beam splitter, beam path ---- Un-shifted (illumination) beam

Shifted (locking)

A further modification to the system was that the multi-mode fibre used in the single component measurements to transport the beams to the light sheet generator, was replaced with a single-mode, polarization-preserving fibre. This was changed to ensure that the spatial profile of both beams remained the same. If these differ significantly, the illumination profiles can also differ, especially when a thick sheet is required, such as when illuminating the face of 
disc; previously ${ }^{7}$ when making single component measurements on the disc it was noted that a 'white card' type correction was needed to correct for this.

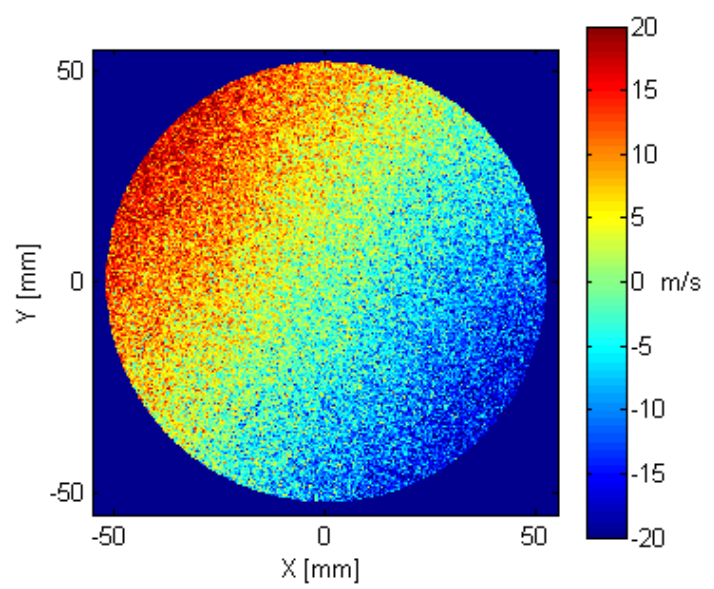

Figure 11. A single measured velocity component of a rotating disc, calculated using the normal sensitivity scheme.

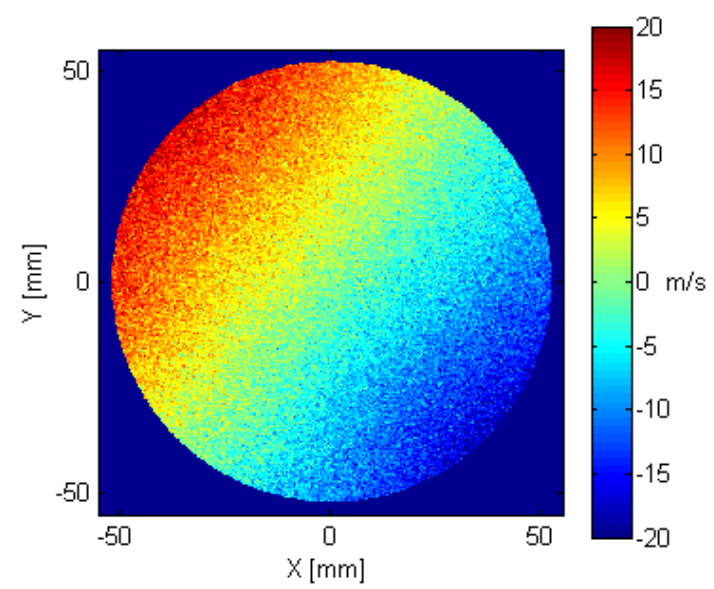

Figure 12. A single measured velocity component of a rotating disc, calculated using the increased sensitivity scheme.

Both the normal and increased sensitivity schemes described above were used to measure the velocity field of a rotating disc. This provides a well-known velocity field with which to characterize the performance of the system. Each arm of the imaging fibre bundle was used with a standard SLR camera lens to view the rotating disc. The disc itself was $200 \mathrm{~mm}$ in diameter, although the common field of view of each observation direction was an approximate disc $100 \mathrm{~mm}$ in diameter. The rotation of the disc was measured using an optical tachometer giving a maximum velocity in the field of view of $\sim \pm 34 \mathrm{~ms}^{-1}$. Each of the observation directions images were processed to yield the measured velocity components, with the direction given by $(\hat{\boldsymbol{o}}-\hat{\boldsymbol{\imath}})$ for that view. Examples of a typical component can be seen in figure 11 and 12 for both the normal sensitivity scheme and increased sensitivity scheme respectively. Here it can be seen that the velocity component captured using the increased sensitivity scheme is visibly less noisy than that captured using the normal sensitivity scheme. 


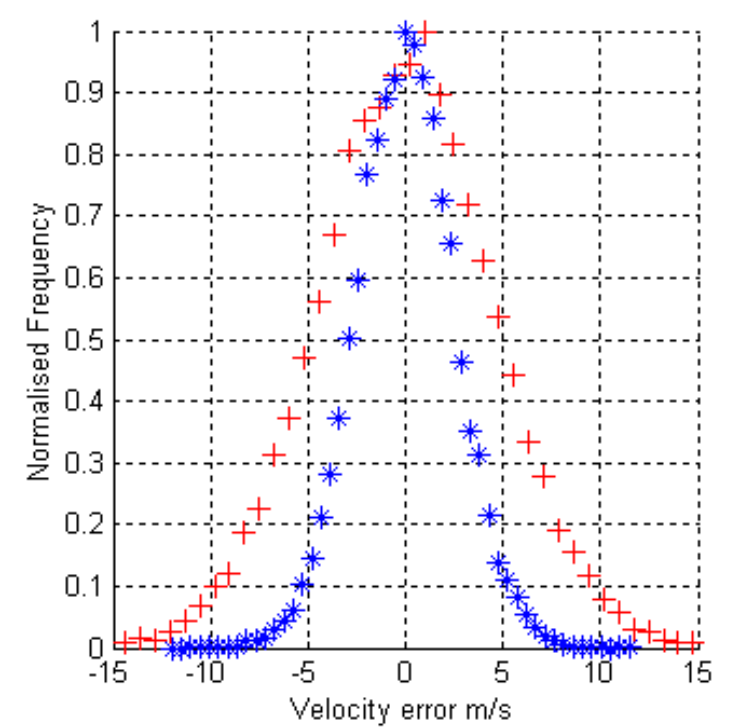

Figure 13. Histograms showing the difference between the measured and theoretical velocity components.

+ Normal sensitivity $2 v$-PDV technique,

* Increased sensitivity $2 v$-PDV technique.

typically $40 \%$ smaller for the increased sensitivity scheme

Table 1. Standard deviations of the variation between the measured and theoretical velocity components, and the calculated reduction in error when using the increased sensitivity scheme.

\begin{tabular}{lllll}
\hline Standard deviation & View 1 & View 2 & View 3 & View 4 \\
\hline Normal sensitivity & $4.6 \mathrm{~ms}^{-1}$ & $4.9 \mathrm{~ms}^{-1}$ & $2.4 \mathrm{~ms}^{-1}$ & $3.9 \mathrm{~ms}^{-1}$ \\
Increased sensitivity & $2.5 \mathrm{~ms}^{-1}$ & $2.9 \mathrm{~ms}^{-1}$ & $1.6 \mathrm{~ms}^{-1}$ & $2.3 \mathrm{~ms}^{-1}$ \\
\% Reduction & $45.7 \%$ & $40.8 \%$ & $33.3 \%$ & $41.0 \%$ \\
\hline
\end{tabular}

The adoption of a single mode fibre for transporting the beam from the two-frequency beam generator to the sheet forming optics, results in identical signal and reference illumination profiles and there being no need to apply the 'white card' correction to realize the velocity field.

Table 2. Standard deviations of the variation between the calculated and theoretical orthogonal velocity components, and the calculated reduction in error when using the increased sensitivity scheme.

\begin{tabular}{|c|c|c|c|}
\hline Standard deviation & $\mathbf{U}$ & $\mathbf{V}$ & $\mathbf{W}$ \\
\hline $\begin{array}{l}\text { Normal sensitivity } \\
\text { Increased sensitivity } \\
\% \text { Reduction }\end{array}$ & $\begin{array}{l}3.6 \mathrm{~ms}^{-1} \\
2.2 \mathrm{~ms}^{-1} \\
38.9 \%\end{array}$ & $\begin{array}{l}6.8 \mathrm{~ms}^{-1} \\
4.2 \mathrm{~ms}^{-1} \\
38.2 \%\end{array}$ & $\begin{array}{l}4.4 \mathrm{~ms}^{-1} \\
2.4 \mathrm{~ms}^{-1} \\
45.5 \%\end{array}$ \\
\hline
\end{tabular}




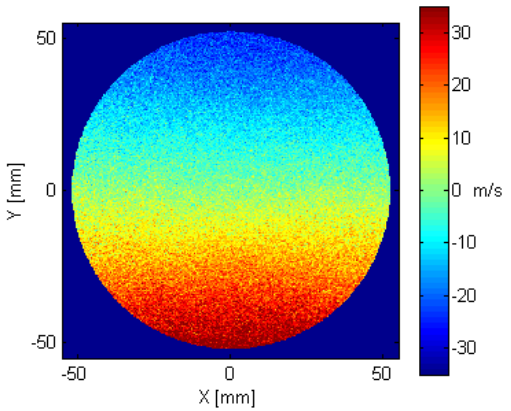

U (normal sensitivity)

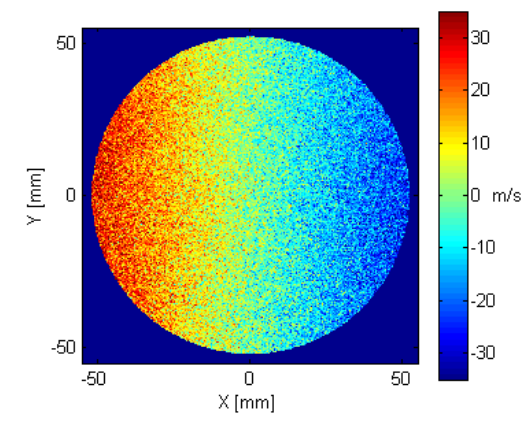

V (normal sensitivity)

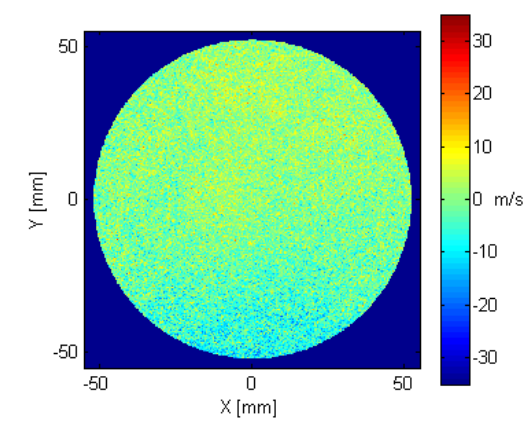

W (normal sensitivity)

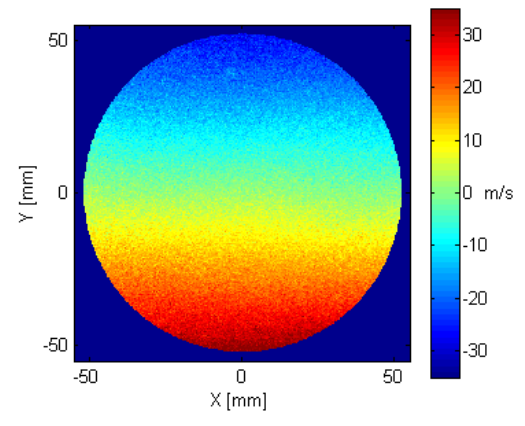

U (increased sensitivity)

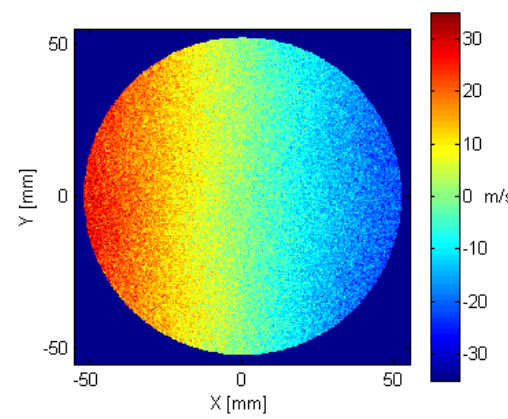

V (increased sensitivity)

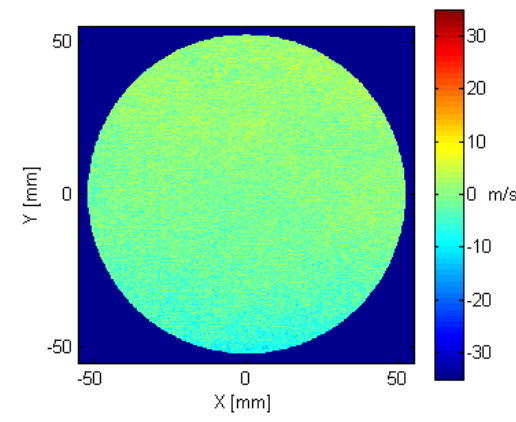

W (increased sensitivity)

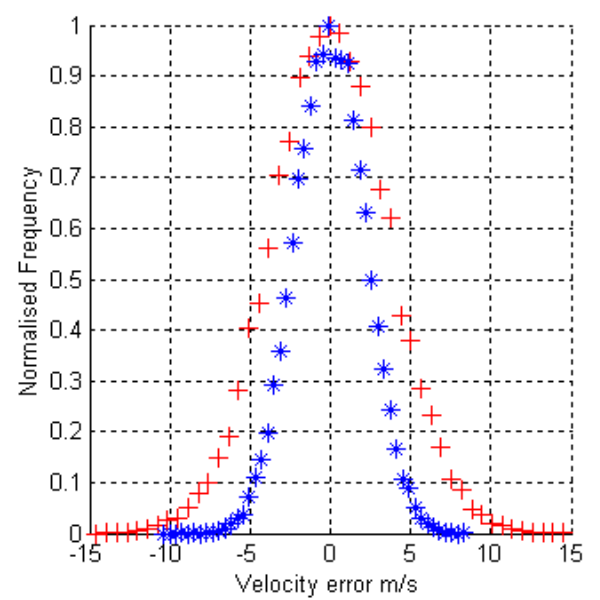

U error

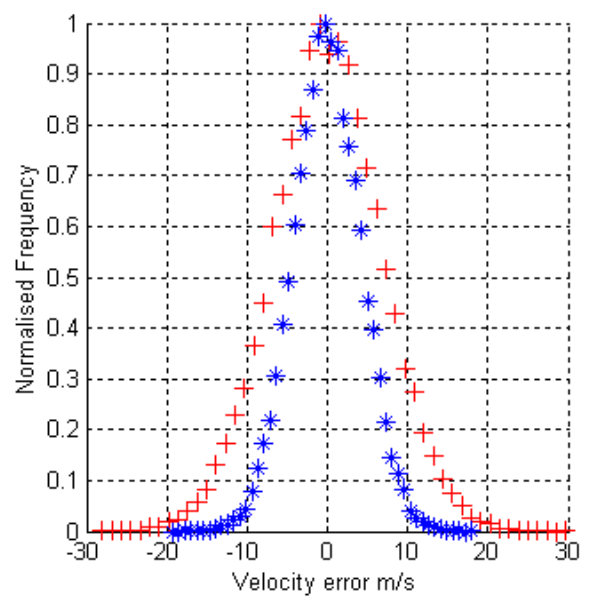

V error

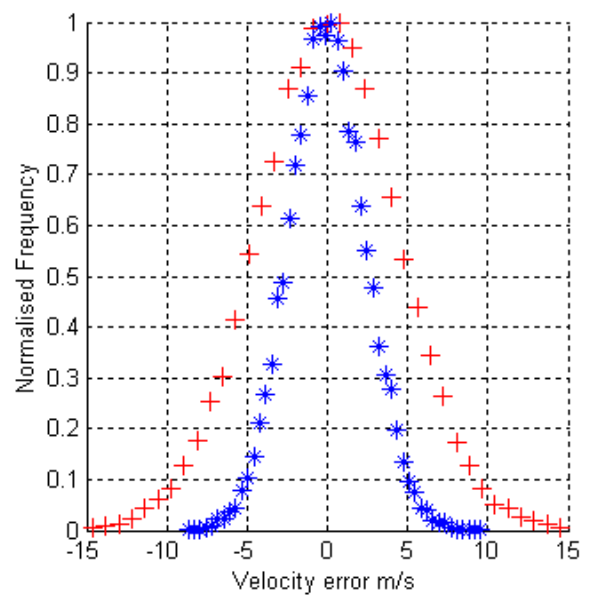

W error

Figure 14. Comparison of computed orthogonal velocity components; (left column) Examples of the calculated orthogonal velocity components using the normal sensitivity $2 v$-PDV technique; (middle column) Examples of the calculated orthogonal velocity components using the increased sensitivity $2 v$-PDV technique; (right column) Histograms showing the difference between the measured and theoretical velocity component. + Normal sensitivity 2v-PDV technique. * Increased sensitivity $2 v$-PDV technique. 
The system was then demonstrated on an axis-symmetric air jet described in section II.A. The system was set-up as shown in figure 15 with three views positioned on one side of the sheet and the fourth viewing from the opposite side. The ability to position views on opposite sides of the light sheet greatly improves the propagation of errors from the measured velocity components to the orthogonal velocity components however this is not possible when making measurements of a rotating disc as the surface of the disc itself defines the light sheet.

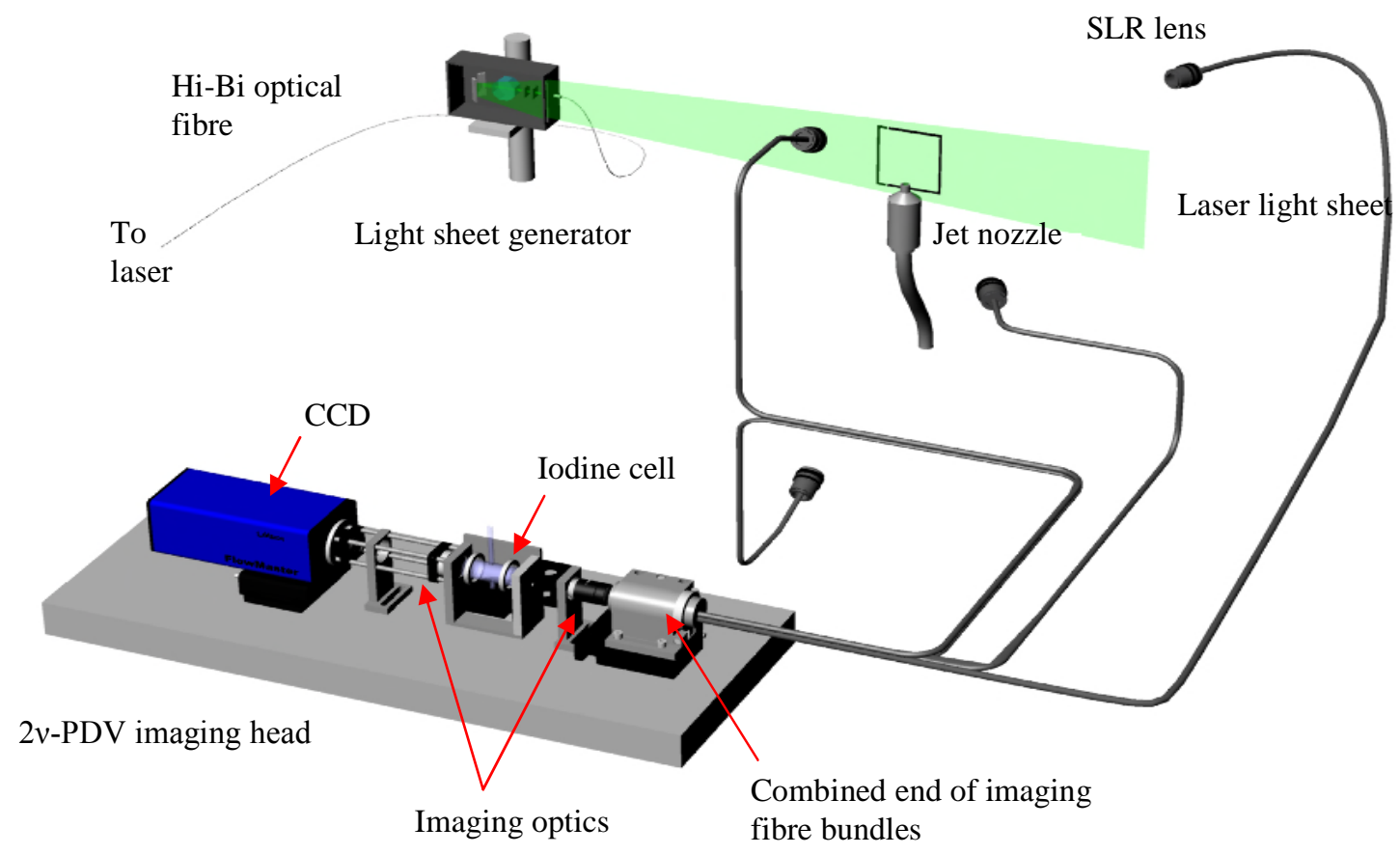

Figure 15. 3D schematic diagram showing the experimental configuration used for measurements on the seeded air jet.

An example of a measurement is shown in figure 16, with vectors showing the in-plane velocities and the colour representing the out-of-plane component. Images were captured using an integration time of ten seconds, and the jet was seeded through the air intake. This resulted in low seeding levels and low scattered light levels for the regions outside of the jet. A threshold has been applied to the data removing these regions of low collected signal. As with the measurements on the rotating disc there was no need to apply a 'white card' correction. It can be seen that the measurements show the expected development of the jet, in the main flow direction, with minimal out-of-plane velocities. 


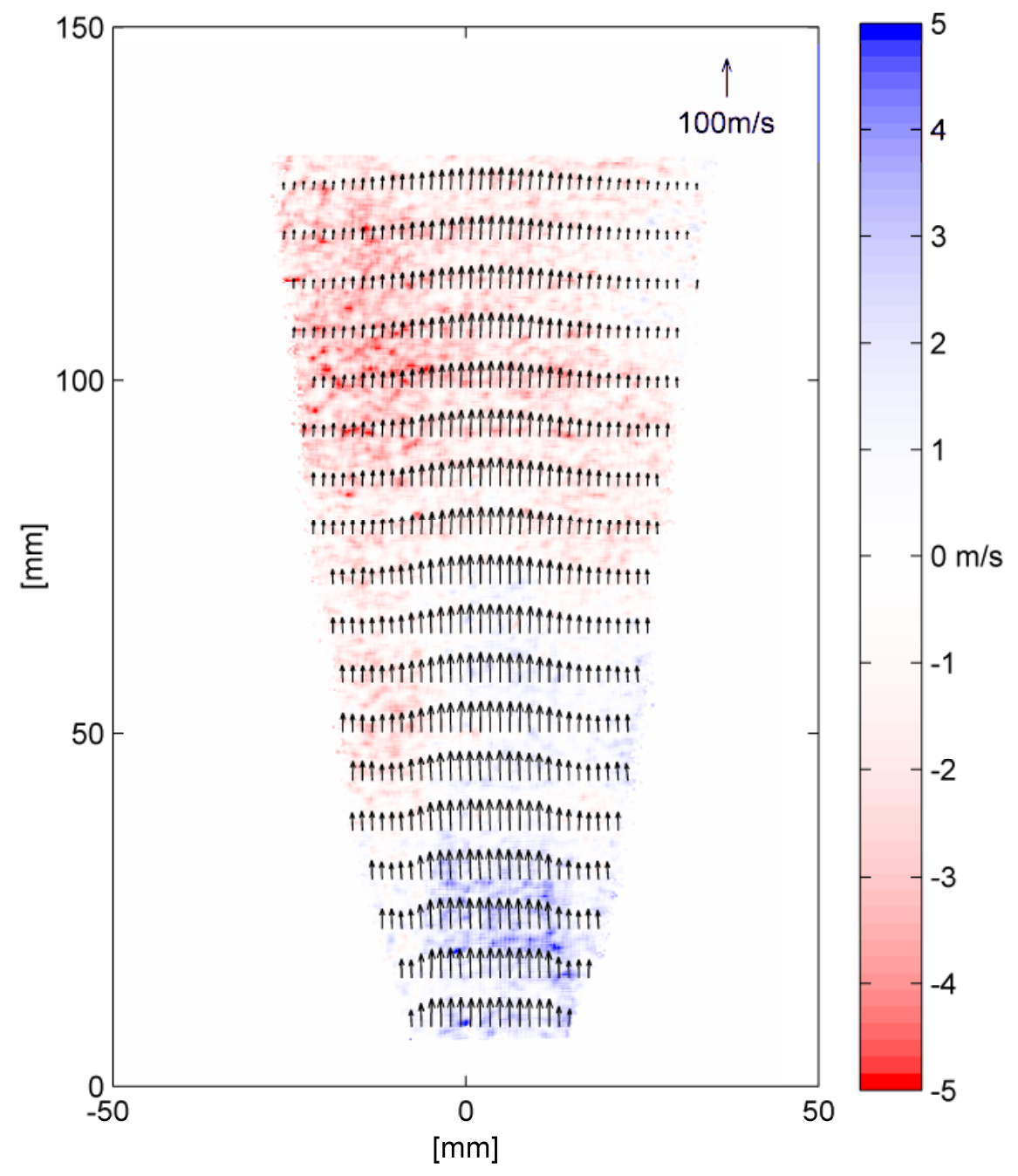

Figure 16. Example of a 3D 2v-PDV measurement made on a seeded air jet. Vectors show the in plane velocities (every $8^{\text {th }}$ and $40^{\text {th }}$ vector show in the horizontal and vertical directions respectively) and colour the out of plane velocity.

A second set of measurements was made with the jet nozzle rotated, so that the main flow was in the out-ofplane direction, a 'swirler' was introduced into the nozzle in an attempt to create more in-plane flow velocities. The nozzle was then positioned at various distances from the sheet and measurements made of the flow velocities in several slices through the jet; this is shown in figure 17 (a). A single slice, at 120mm downstream from the nozzle, is shown in figure 17 (b). Again in these measurements the vectors represent the in-plane velocity components and the colour represents the out-of-plane velocity component. Regions of low seeding and scattered light intensity have been removed using a threshold. It can be seen, in figure 18, that the jet was swirling and drawing in air predominantly from one side of the flow, this could also been seen visually in the light sheet. 


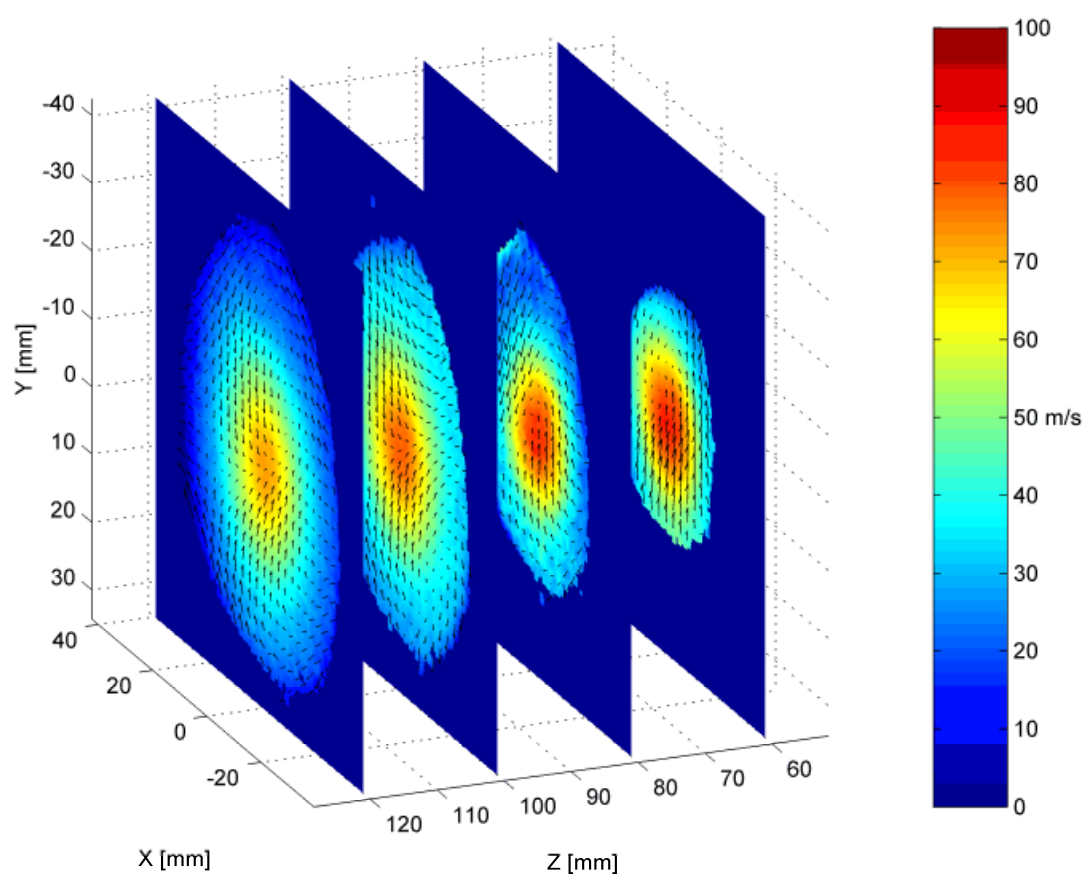

(a)

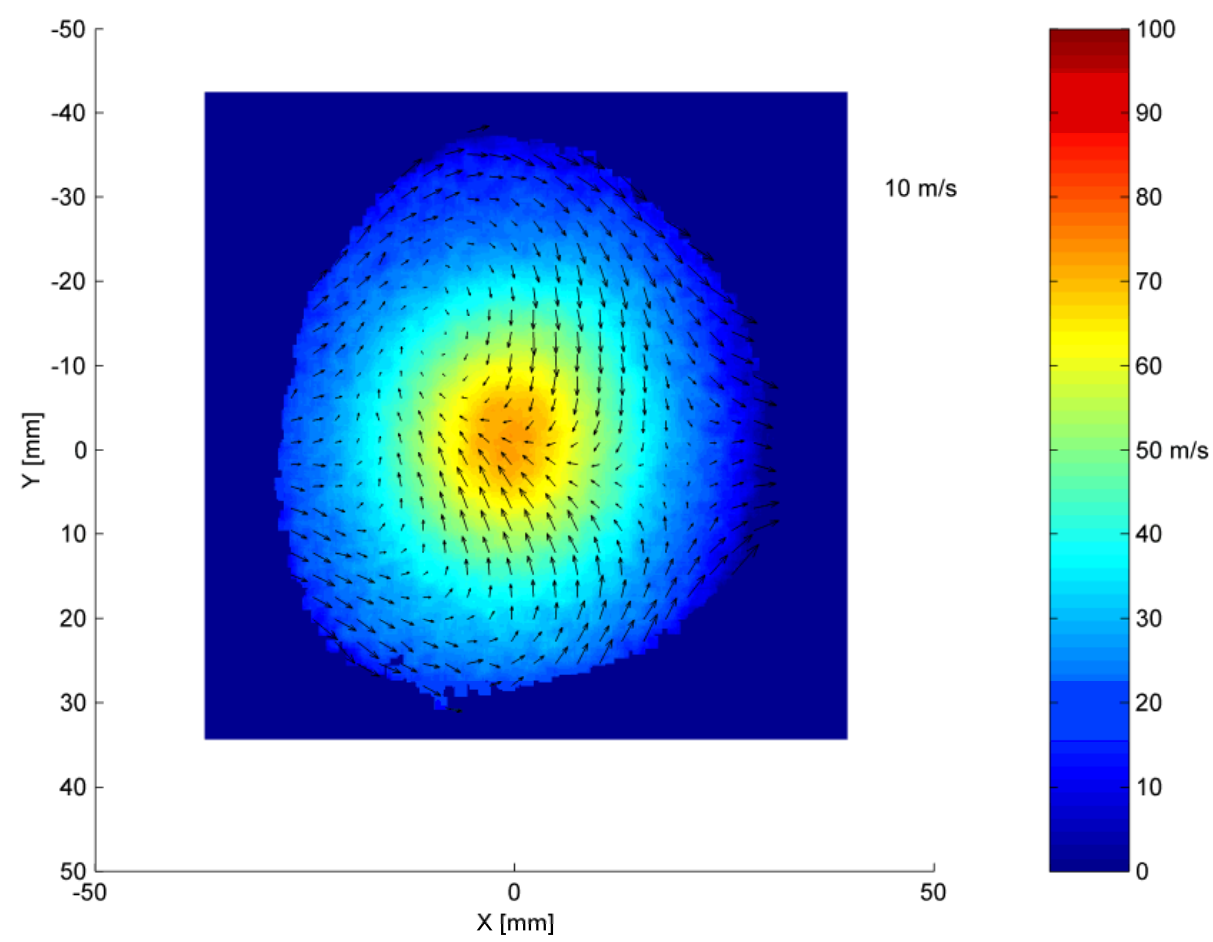

(b)

Figure 17. 3D 2v-PDV cross section measurements on a seeded air jet. (a) Cross sections measured at $60,80,100$ and $120 \mathrm{~mm}$ from the jet nozzle. (b) Cross section at $120 \mathrm{~mm}$ downstream from the nozzle exit. Vectors represent the in plane velocities (every $12^{\text {th }} \times 12^{\text {th }}$ vector shown) and colour the out-of-plane velocity. 


\section{Discussion and Conclusions}

A PDV technique has been developed that reduces the number of CCD cameras required in a PDV imaging head from two to one. The $2 v$-PDV technique has been successfully applied to make time-averaged single velocity component measurements and with the addition of the multiple branch imaging fibre bundles, time-averaged 3D velocity measurements.

The combination of the $2 v$-PDV technique with the imaging fibre bundles allows $3 \mathrm{D}$ velocity measurements to be made using only a single camera and iodine cell, greatly reducing the cost and complexity associated with conventional 3D PDV techniques which require six cameras and three iodine cells for $3 \mathrm{~d}$ measurements. The use of the imaging fibre bundles also allows greater flexibility in the positioning of the observation directions and can remove problems associated with isolating the iodine cells and cameras from the environment.

As both images are now captured on the same camera the pixel-matching problem that affects conventional PDV is avoided. Because $2 v$-PDV does not suffer from this problem and there being no differences in the optical paths for the two images, the commonly used 'white card' correction is also no longer needed in the processing to realize the velocity field.

However the images used in the normalization process are now captured sequentially, unlike in conventional PDV when they are captured simultaneously. For steady-state flows this should not be a problem, provided that the seeding is relatively dense and the seeding distribution remains essentially unchanged during the time taken to acquire the two images. However this will not be the case for some flows. In a rapidly time-varying flow, averaging over long integration times is no longer an option so a pulsed laser $2 v-\mathrm{PDV}$, to freeze the motion of the particles is proposed. An investigation of this is currently underway.

The ability to improve the sensitivity of the PDV measurements by positioning the two laser frequencies on either side of the iodine absorption line has been demonstrated. Using measurements made on a rotating disc a typical reduction in the error of approximately $40 \%$ can be achieved using this increased sensitivity method.

\section{Acknowledgments}

This work was funded by the Engineering and Physical Sciences Research Council (EPSRC), UK (GR/SO4291).

\section{Reference List}

1. Komine, H., Brosnan, S., Litton, A., and Staepperts, E., "Real-Time Doppler Global Velocimetry", AIAA 29th Aerospace Sciences Meeting, Reno, Nevada, Paper 91-0337, 1991.

2. Meyers, J.F. and Lee, J.W., "Proof of concept test of the Doppler Global Velocimeter", Spaceborne Photonics: Aerospace Applications Of Lasers And Electro-optics, Newport Beach, CA, 1991.

3. Meyers, J.F., Lee, J.W., and Schwartz, R.J., "Characterization of Measurement Error Sources in Doppler Global Velocimetry", Measurement Science and Technology, 12, 357-368, 2001.

4. Ford, H.D. and Tatam, R.P., "Development of Extended Field Doppler Velocimetry for Turbomachinery Applications", Optics and Lasers in Engineering, 27, 675-696, 1997.

5. Nobes, D.S., Ford, H.D., and Tatam, R.P., "Three Component Planar Doppler Velocimetry Using Imaging Fibre Bundles", Experiments in Fluids, 36, 1, 3-10, 2004.

6. Gerstenkorn, S. and Luc, P., Atlas du Spectre d'Absorption de la Molecule d'Iode 14800-200 $\mathrm{cm}^{-1}$ Complement: Identification des Transitions du Systeme (B-X),Editions du Centre Nationale de la Recherche Scientifique, Paris, France, 1986

7. Charrett, T.O.H., Ford, H.D., Nobes, D.S., and Tatam, R.P., "Two frequency Planar Doppler Velocimetry (2v-PDV)", Review of Scientific Instruments, 75, 11, 4487-4496, 2004.

8. Charrett, T.O.H. and Tatam, R.P., "Single camera three component planar velocity measurements using two frequency Planar Doppler Velocimetry (2n-PDV) ", Measurement Science and Technology, (In press).

9. Thorpe, S.J., Ainsworth, R.W., and Manners, R.J., "The Development of a Doppler Global Velocimeter and its Application to a Free Jet Flow", ASME / JSME Fluids Engineering and Laser Anemometry Conference and Exhibition, Hilton Head, SC, USA, 1995. 
10. Ainsworth, R.W. and Thorpe, S.J., "The Development of a Doppler Global Velocimeter for Transonic Turbine Applications", International Gas Turbine and Aeroengine Congress and Exposition, The Hague, Netherlands, ASME 94-GT146, 1994.

11. Arnette, S.A., Elliott, G.S., and Mosedale, A.D., "Two-colour planar Doppler Velocimetry", AIAA Journal, 38, 11, 2001-2006, 2000.

12. Muller, H., Eggert, M., Pape, N., Dopheide, D., Czarske, J., Buttner, L., and Razik, T., "Time resolved DGV based on laser frequency modulation", 12th International symposium on the application of Laser Techniques to fluids, Lisbon, Portugal., 2004.

13. Roehle, I., Willert, C., Schodl, R., and Voigt, P., "Recent Developments and Applications of Quantitative Laser Light Sheet Measuring Techniques in Turbo machinery Components", Measurement Science and Technology, 11, 1023-1035, 2000.

14. Nobes, D.S., Wieneke, B., and Tatam, R.P., "Determination of View Vectors from Image Warping Mapping Functions", Optical Engineering, 43, 2, 407-414, 2004.

15. Willert, C., Stockhausen, G., Beversdorff, M., klinner, J., Lempereur, C., Barricau, P., Quest, J., and Jansen, U., "Application of Doppler Global velocimetry in cryogenic wind tunnels", Experiments in Fluids, 39, 420-430, 2005. 
2006

Single camera 3D planar Doppler

velocity measurements, using two

frequency planar Doppler velocimetry

(2v-PDV) and imaging fibre bundles

Charrett, Thomas O. H.

AIAA

Charrett, T.O, Ford, H.D. and Tatam, R.P. 2006. Single camera 3D planar Doppler velocity measurements, using two frequency planar Doppler velocimetry (2v-PDV) and imaging fibre bundles. In Proceedings of the 44th AIAA aerospace sciences meeting and exhibit (MASM06),

9-12th January 2006, Reno, NV, USA, paper number AIAA 2006-47. Reston, VA: AIAA. DOI: http://dx.doi.org/10.2514/6 http://dx.doi.org/10.2514/6.2006-47

Downloaded from Cranfield Library Services E-Repository 